\title{
TRIM58/cg26157385 methylation is associated with eight prognostic genes in lung squamous cell carcinoma
}

\author{
WEIMIN ZHANG $^{1 *}$, QICHEN CUI $^{1 *}$, WEIFENG QU $^{1}$, XIAOYUN DING $^{1}$, \\ DONGLIN JIANG ${ }^{2}$ and HONGCHENG LIU ${ }^{3}$
}

\begin{abstract}
Departments of ${ }^{1}$ Thoracic Surgery and ${ }^{2}$ Central Laboratory, The Third Affiliated Hospital of Nantong University, Wuxi, Jiangsu 214000; ${ }^{3}$ Department of Thoracic Surgery, Shanghai Pulmonary Hospital, Shanghai 200000, P.R. China
\end{abstract}

Received October 26, 2017; Accepted March 30, 2018

DOI: $10.3892 /$ or.2018.6426

\begin{abstract}
The present study aimed to analyze the differentially expressed genes related to the tripartite motif containing 58 (TRIM58)/cg26157385 methylation sites, and consequently to provide theoretical basis for elucidating the influence of TRIM58/cg26157385 methylation on lung cancer prognosis. Methylation-sequencing information, mRNA expression profiling data and clinical data were downloaded from cBioPortal database to screen out candidate genes related to the methylation of TRIM58/cg26157385 in squamous cell lung carcinoma. The differentially expressed genes related to TRIM58 methylation were extracted form both training dataset and validation dataset. Cox regression analysis, risk scoring system construction, correlation analysis between the expression value of genes and clinical information were conducted to reveal TRIM58 methylation-related factors. Additionally, GO function analysis and KEGG pathway enrichment analysis were performed. Based on their expression level and the corresponding survival information for 347 out of 370 samples with squamous cell lung carcinoma, 183 genes significantly associated with prognosis were gained, and the top 8 ones, including alpha-2-macroglobulin-like 1 (A2ML1), cyclin-E1 (CCNE1), COBL, establishment of sister chromatid cohesion $\mathrm{N}$-acetyltransferase 2 (ESCO2), G protein-coupled receptor 115 (GPR115), matrix metalloproteinases 10 (MMP10), OVO homologue-like 1 (OVOL1) and secretoglobin family 1A member 1 (SCGB1A1), were candidate signature genes significantly correlated with TRIM58 methylation. Furthermore, targeted therapy was significantly correlated with prognosis. Functional enrichment analysis demonstrated
\end{abstract}

Correspondence to: Dr Weimin Zhang, Department of Thoracic Surgery, The Third Affiliated Hospital of Nantong University, 558 Xingyuan North Road, Wuxi, Jiangsu 214000, P.R. China

E-mail: zhangweiminwx@sina.com

*Contributed equally

Key words: squamous cell lung carcinoma, TRIM58, methylation, functional enrichment analysis that the proliferation and differentiation of epidermal cells in lung squamous cell carcinoma patients were abnormal and the homeostasis was disturbed. Eight genes, including A2ML1, CCNE1, COBL, ESCO2, GPR115, MMP10, OVOL1 and SCGB1A1, were significantly related to TRIM58 methylation and treatment of lung squamous cell carcinoma, and may be used as potential prognostic biomarkers. The present study would help to elucidate the influence of TRIM58/cg26157385 methylation on lung cancer prognosis.

\section{Introduction}

Lung cancer is the most common cancer and the leading cause of cancer-related deaths in both men and women in the USA and China, among which the proportion of squamous cell lung carcinoma was as high as $30-40 \%(1,2)$. As a common type of lung cancer, the histological changes of squamous cell lung carcinoma have been basically identified, which mainly included the loss of cilia, squamous metaplasia, atypical hyperplasia and dysplasia induced by chronic stimulation, and injury of the bronchial mucosa columnar epithelium (3). Research on etiology and pathogenesis has confirmed that the occurrence and development of squamous cell lung cancer is a pathological process involving multiple genes and pathways. However, genomic alterations in squamous cell lung cancers have not been comprehensively characterized and no molecular-targeted agents have been specifically developed for its treatment (4). Therefore, it is urgently needed to elucidate the mechanisms underlying the occurrence and development of squamous cell lung cancer and develop new therapeutic targets.

Among numerous tumor pathogenesis, methylation of the functional genes has attracted the interest of many researchers. Aberrant promoter island methylation of tumor suppressor genes, including overall low level methylation and hyper-methylation in some local regions, has been established as a common epigenetic mechanism underlying the pathogenesis of human cancers (5-7). The tripartite motif (TRIM) proteins are a family of proteins with conserved structure, rapid evolution and E3 ubiquitin ligase activities. TRIM is involved in a broad range of cellular processes and diseases, including innate immunity, development process, genetic diseases and cancer (8). Thus far, more than 60 members of the TRIM family proteins have been found in humans (9), among 
which, the tripartite motif containing 58 (TRIM58) was identified as a candidate tumor suppressor and a novel methylated gene $(7,10)$. TRIM58 has been reported to regulate terminal erythroid cell cycles and enucleation (11), and the overexpression of TRIM58 has been associated with the prognosis of early lung cancer (12). Furthermore, aberrant inactivation of TRIM58 consequent to CpG island hyper-methylation may stimulate early carcinogenesis of lung adenocarcinoma (7). However, further functional research and verification on the association between TRIM58 and the prognosis of lung cancer are still needed.

In the present study to elucidate the influence of TRIM58/cg26157385 methylation on lung cancer prognosis, we used the large quantities of mRNA-Seq data in lung squamous cell carcinoma patients published in cBioPortal database (http://www.cbioportal.org/), to screen out candidate genes related to the methylation of TRIM58/cg26157385, and construct a prognostic discrimination system based on these genes. The reliability of the prognostic discrimination system was further validated in an independent dataset.

\section{Materials and methods}

Data analysis. The data analysis flow of the present study is briefly summarized in Fig. 1.

Collection and pretreatment of collected data. On February 22, 2017, two sets of squamous cell lung carcinoma data were downloaded from cBioPortal database (http://www.cbioportal.org/). One dataset was selected as the training dataset, in which there were 370 squamous cell lung carcinoma samples with both methylation sequencing information (the platform was Illumina Methylation450) and mRNA expression profiling data (the platform was IlluminaHiSeq2000). The other dataset was selected as the validation dataset, including 178 squamous cell lung carcinoma samples with mRNA expression profiling data (the platform was IlluminaHiSeq2000). Furthermore, 51 normal control samples were obtained from the cBioPortal database.

\section{Clinical data}

Information of samples in the training dataset. The 370 patients with squamous cell lung carcinoma consisted of 267 males $(74.17 \%)$ and 93 females $(25.83 \%)$ with mean age of $67.71 \pm 8.65$ years. There were 114 smokers $(32.11 \%)$, 13 non-smokers $(3.66 \%)$ and 228 cases $(64.23 \%)$ who had quit smoking. The number of patients who received radiotherapy and those who had not received radiotherapy was $36(11.92 \%)$ and $266(88.08 \%)$, respectively. The number of patients who received targeted therapy and those who had not received targeted therapy was $93(30.69 \%)$ and 210 (69.31\%), respectively, with a mean survival time of $26.99 \pm 28.92$ months. A total of 135 patients died at the end of the follow-up period, accounting for $33.24 \%$ of the total population.

Information of samples in the validation dataset. The 178 patients with squamous cell lung carcinoma consisted of 133 males $(74.72 \%)$ and 45 females $(25.28 \%)$ with mean age of $67.86 \pm 8.25$ years. There were 27 smokers $(16.56 \%)$, 4 non-smokers $(2.45 \%)$ and 132 cases $(80.98 \%)$ who had quit

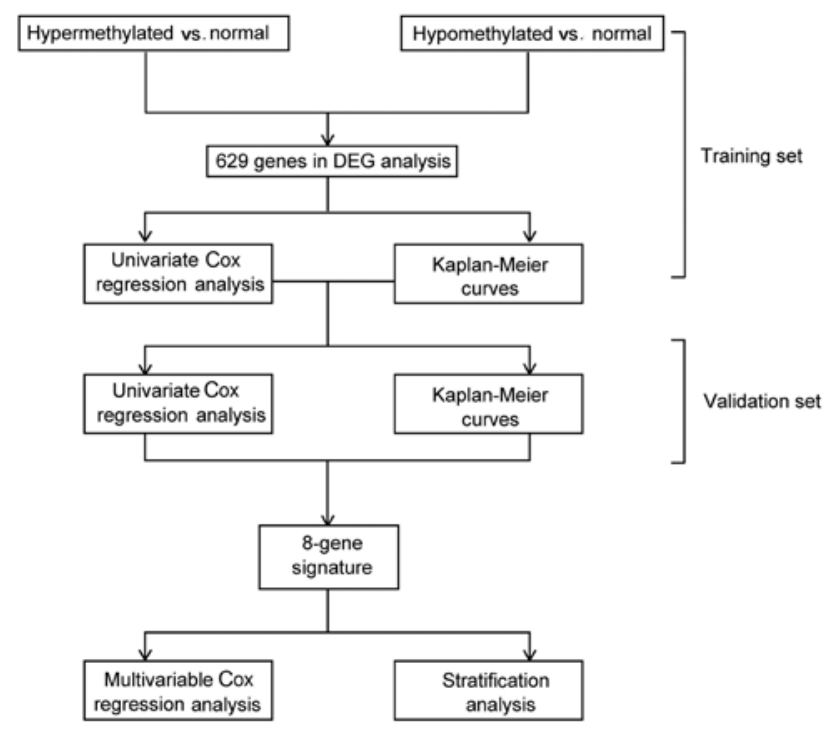

Figure 1. Data analysis flow.

smoking. The number of patients who received radiotherapy and those who had not received radiotherapy was $14(12.5 \%)$ and $98(87.5 \%)$, respectively. The number of patients who received targeted therapy and those who had not received targeted therapy was $35(30.43 \%)$ and $80(69.57 \%)$, respectively, with mean survival time of $29.96 \pm 31.85$ months. A total of 78 patients died at the end of the follow-up period, accounting for $43.82 \%$ of the total population.

Information of normal control samples. The 51 patients in the normal control samples consisted of 35 males $(68.63 \%)$ and 16 females $(31.37 \%)$ with mean age of $68.34 \pm 8.49$ years. There were 13 smokers $(25.49 \%), 34$ cases $(66.67 \%)$ who had quit smoking and 4 cases $(7.84 \%)$ without any information. The number of patients who received radiotherapy, those who had not received radiotherapy and the patients without any information was 3 (5.88\%), 35 (68.63\%) and 13 (25.49\%), respectively. The number of patients who received targeted therapy, who had not received targeted therapy, and those without any information was 13 (25.49\%), 25 (49.02\%) and 13 (25.49\%), respectively, with mean survival time of $23.93 \pm 27.97$ months. A total of 27 subjects died at the end of the follow-up period, accounting for $52.94 \%$ of the total population. The information of samples in the training, validation and normal control dataset is listed in Table I.

Grouping of methylation samples. DNA methylation $\beta$-values are continuous variables between 0 (completely unmethylated) and 1 (completely methylated), representing the ratio of the intensity of the methylated bead type to the combined locus intensity (13). The methylation data of TRIM58/cg26157385 in each sample were included in the Illumina Methylation450 platform, which consisted of two peaks, 0.1 and 0.9 , corresponding to the low and high degree of methylation, respectively. As higher $\beta$-values represent higher level of DNA methylation, we divided the samples into low-methylation samples with $\beta$-values of 0.1-0.5 and high-methylation samples with $\beta$-values of 0.5-0.9. Finally, 133 high-methylation samples and 237 low-methylation samples were obtained. Furthermore, 
Table I. Clinical information of the patients in the training and the validation datasets.

\begin{tabular}{lccc}
\hline & Training dataset & Validation dataset & Normal control dataset \\
\hline Sample count & 370 & 178 & 51 \\
Sex (Male/female) & $267 / 93$ & $133 / 45$ & $35 / 16$ \\
Age (mean \pm SD) & $67.71 \pm 8.65$ & $67.86 \pm 8.25$ & $68.34 \pm 8.49$ \\
Smoking status (Yes/reform/no/NA) & $114 / 228 / 13 / 15$ & $27 / 132 / 4 / 15$ & $13 / 34 / 0 / 4$ \\
Radiotherapy (Yes/no/NA) & $36 / 266 / 68$ & $14 / 98 / 66$ & $3 / 35 / 13$ \\
Targeted-therapy (Yes/no/NA) & $93 / 210 / 67$ & $35 / 80 / 63$ & $13 / 25 / 13$ \\
Death (Yes/no) & $135 / 235$ & $78 / 100$ & $27 / 24$ \\
Overall survival months (mean \pm SD) & $26.99 \pm 28.92$ & $29.96 \pm 31.85$ & $23.93 \pm 27.97$ \\
\hline
\end{tabular}

Reform means the patients had quit smoking. NA, data unavailable.

there were significant differences of the $\beta$-values between all the tumor samples and the normal control samples (Fig. 2).

Screening of differentially expressed genes. The mRNA expression profiling data corresponding to the high and low degrees of methylation samples in the training dataset were analyzed for the differentially expressed genes using the limma and multtest package (http://bioconductor. org/packages/release/bioc/html/multtest.html) in R language version 3.0.1 (14). FDR value $<0.05$ and fold change value $>1.5$ were selected as threshold.

Screening of genes significantly related to prognosis and calculation of risk score. There were corresponding survival information for 347 out of 370 samples with squamous cell lung carcinoma. Screening of genes significantly related to prognosis was performed using Cox regression analysis and the significant $\mathrm{P}$-value and the prognostic correlation coefficient $\beta$-value were calculated using the log-rank test. According to the prognostic correlation coefficient $\beta$-value and the expression value of the gene, the risk score computing model was defined as follows:

Risk score $=\beta_{\text {gene } 1} \times \operatorname{expr}_{\text {gene } 1}+\beta_{\text {gene } 2} \times$ expr $_{\text {gene } 2}+\cdots+\beta_{\text {genen }} \times$ expr $r_{\text {genen }}$

The prognostic correlation coefficient $\beta$-value was indicated by $\beta$ and the expression value of the gene was indicated by expr. The corresponding risk score value of each sample was calculated and the samples were divided into the high-risk and the low-risk group according to the risk score median which was set as the boundary (15-17).

Correlation analysis of the risk score with the clinical features. Correlation analysis of the risk score with the clinical features, including age $(\geq 60$ and $<60)$, sex (male and female), radiotherapy (yes or no), targeted therapy (yes or no) were performed using the univariate and multivariable Cox regression analyses for survival package. The screened clinical features significantly associated with prognosis were analyzed using the Kaplan-Meier survival curve.

Verification of the risk scoring system. The expression values of signature genes were first extracted from the validation dataset. The corresponding risk score values of each sample were also

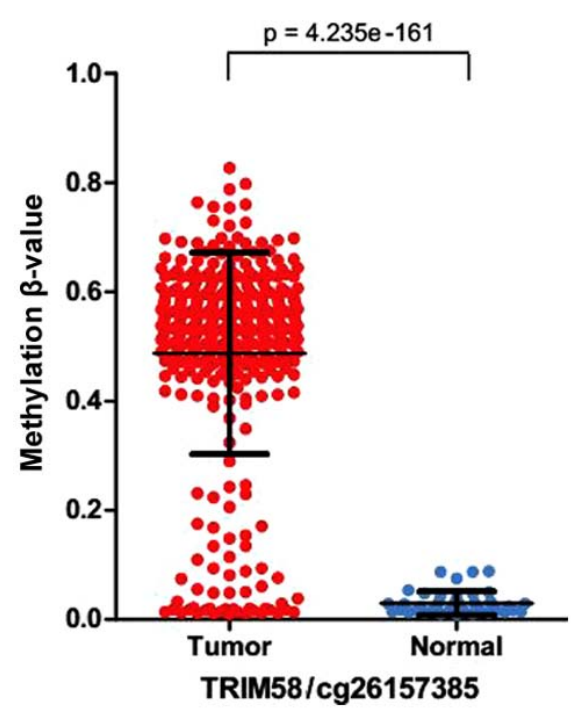

Figure 2. $\beta$-value comparison between the tumor samples and the normal control samples.

calculated according to the expression value of each gene and the samples were divided into the high-risk group and the low-risk group using the risk score median as boundary. The relationship between the high-risk and the low-risk samples and the clinical features were verified using the risk scoring system.

GO analysis and pathway enrichment. The biological process and KEGG pathway enrichment analysis of the selected prognostic genes were performed using the Fisher algorithm using the clusterProfiler package in $\mathrm{R}$ language (18). The specific algorithm is as follows:

$$
p=1-\sum_{i=0}^{x-1} \frac{\left(\begin{array}{l}
M \\
i
\end{array}\right)\left(\begin{array}{l}
N-M \\
K-i
\end{array}\right)}{\left(\begin{array}{l}
N \\
K
\end{array}\right)}
$$

$\mathrm{N}$ represents the total number of genes in the genome, $\mathrm{M}$ represents the number of genes in the pathway and $\mathrm{K}$ indicates the number of differentially expressed genes. The Fisher's 
score indicates the ratio of genes (number X) belonging to the functional pathway out of the total differentially expressed genes (number K).

\section{Results}

Detection and validation of eight prognostic genes associated with TRIM58/cg26157385 methylation. Except the death numbers and the overall survival months, there were no significant differences among the clinical features between the samples in the training set and the samples in the normal controls (Table II). Compared with the 51 samples in the normal group, there were 527 and 449 significantly different expression genes in the 133 high-methylation samples and 237 low-methylation samples, respectively. A total of 629 significantly differentially expressed genes related to TRIM58 methylation were screened.

Based on the expression level of these 629 genes and the corresponding survival information of the tumor samples, 183 genes significantly associated with prognosis $(\mathrm{P}<0.05)$ were screened using the Cox regression analysis. Eight genes with $\mathrm{P}$-value $>10^{-5}$ were selected as candidate signature genes, including alpha-2-macroglobulin-like 1 (A2ML1), cyclin-E1 (CCNE1), COBL, establishment of sister chromatid cohesion $\mathrm{N}$-acetyltransferase 2 (ESCO2), G protein-coupled receptor 115 (GPR115), matrix metalloproteinases 10 (MMP10), OVO homologue-like 1 (OVOL1) and secretoglobin family $1 \mathrm{~A}$ member 1 (SCGB1A1).

According to the prognostic correlation coefficient $\beta$-value (Table III) and the expression value of genes in each sample, the corresponding risk score of each sample was calculated as follows:

Risk score $=0.659 \times e x p r_{A 2 M L 1}+0.823 \times e x p r_{C O B L}+1.355 \times e x p r_{\text {OVOL1 }}$

$$
\begin{aligned}
& +1.502 \times \exp _{\mathrm{CCNE} 1}+0.442 \times \exp _{\mathrm{MMP} 10}+0.411 \\
& \times \operatorname{expr}_{\mathrm{SCGB} 1 \mathrm{~A} 1}-2.815 \times e x p r_{\mathrm{ESCO} 2}-0.941 \times e \operatorname{expr}_{\mathrm{GPR} 115}
\end{aligned}
$$

The samples in the training dataset were divided into the high-risk group and the low-risk group according to the risk score, and the screened clinical features significantly associated with prognosis were analyzed using the Kaplan-Meier survival curve (Fig. 3A). The results indicated that the risk scoring system successfully classified different samples with different prognosis $(\mathrm{P}=0.0218)$.

Among the 178 samples in the validation dataset, 168 had survival information. The overall survival analysis result was similar to the result of the training dataset $(\mathrm{P}=0.0424)$ (Fig. 3B).

In the training dataset, the median overall survival time of the high-risk group and the low-risk group was 450 and 608 days, respectively, and in the validation dataset, the median overall survival time of the high-risk group and the low-risk group were 460 and 665 days, respectively.

Clinical and molecular characteristics of patients with high and low risk of lung squamous cell carcinoma driven by TRIM58 methylation. The expression values of eight signature genes in different sets of the training dataset are displayed in Fig. 4. Risk score, overall survival days, expression value of

Table II. Comparison of the clinical features between samples in the training dataset and samples in the normal control.

P-value

Sex

Age (years)

Smoking status

Radiotherapy

Targeted-therapy

Death

Overall survival months

${ }^{\mathrm{a}}$ Chi-square test; ${ }^{\mathrm{b}} \mathrm{T}$-test.

Table III. The P-value and the $\beta$-value of signature genes.

\begin{tabular}{lcr}
\hline Gene & P-value & $\beta$-value \\
\hline A2ML1 & $9.56 \mathrm{E}-08$ & 0.659 \\
COBL & $2.30 \mathrm{E}-07$ & 0.823 \\
OVOL1 & $2.49 \mathrm{E}-07$ & 1.355 \\
CCNE1 & $1.49 \mathrm{E}-06$ & 1.502 \\
MMP10 & $2.02 \mathrm{E}-06$ & 0.442 \\
SCGB1A1 & $7.20 \mathrm{E}-06$ & 0.411 \\
ESCO2 & $7.44 \mathrm{E}-06$ & -2.815 \\
GPR115 & $7.93 \mathrm{E}-06$ & -0.941 \\
\hline
\end{tabular}

eight signature genes and clinical information of the corresponding samples in the training dataset and validation dataset are displayed in Fig. 5.

Eight significant expression factors associated with treatment. As displayed in Table IV, the results of single factor Cox regression demonstrated that radiotherapy, targeted therapy and risk score were significantly related to prognosis and the results of further multivariate Cox regression analysis indicated that targeted therapy and risk score were significantly correlated with the prognosis of lung squamous cell carcinoma.

Further survival analysis of two clinical features, targeted therapy and risk score, were performed in the training dataset. As displayed in Fig. 6A-a, the survival rate of high risk-targeted therapy group was significantly lower than the unmethylated samples, while that of low-risk-targeted therapy group was significantly higher than the unmethylated samples. No similar results were observed in the non-therapy group (Fig. 6A-b), which further confirmed the relationship between the targeted therapy and prognosis. Based on the results in the training dataset, the data of the targeted-therapy group corresponding to the validation dataset were collected and the correlation between the clinical information in the high-risk and the low-risk group was verified by the results of the survival rate. As displayed in Fig. 6B-a, the results were consistent with the training set, and the survival rate of 
A

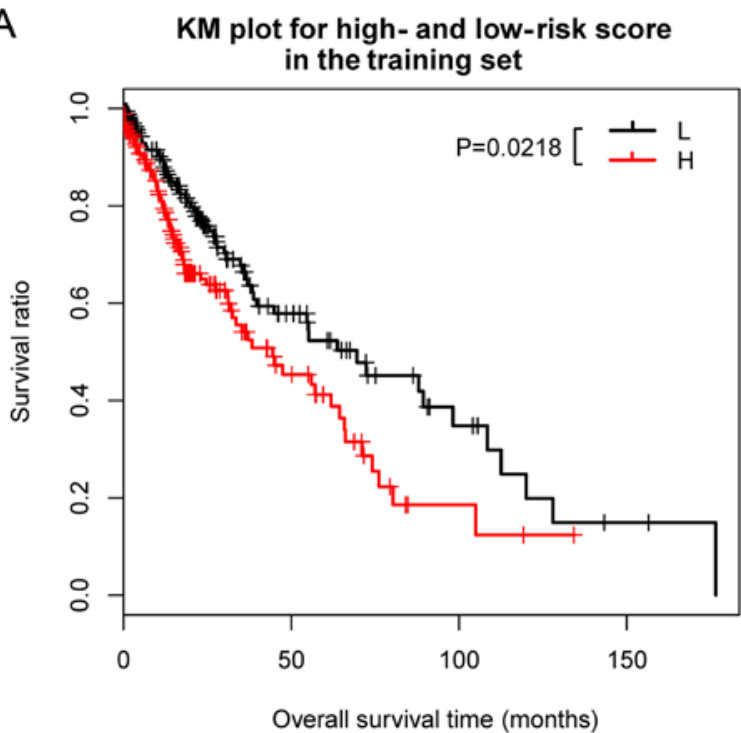

B

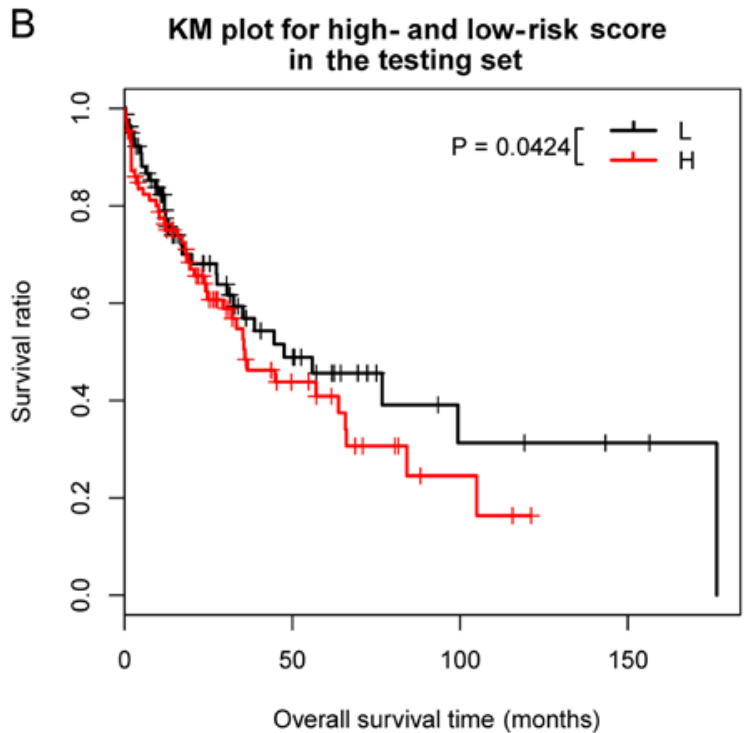

Figure 3. Kaplan-Meier survival curve of high-risk and low risk group samples. (A) The curve in the training dataset. (B) The curve in the validation dataset. The red curve and the black curve indicate the survival curve of patients in the high-risk group and the low-risk group, respectively. $\mathrm{P}=0.0218$, indicated that the P-value of the log-rank test was significant.

A
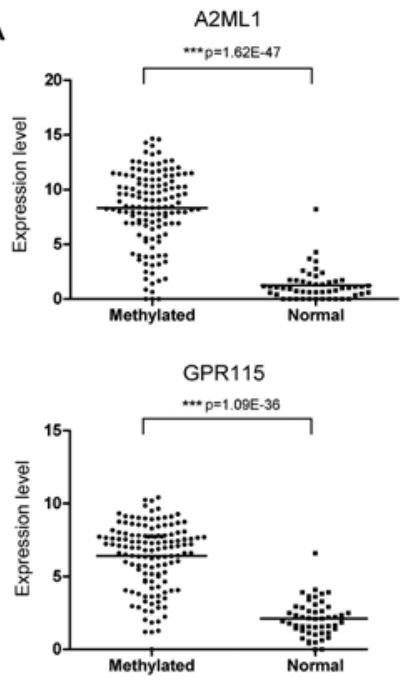

B
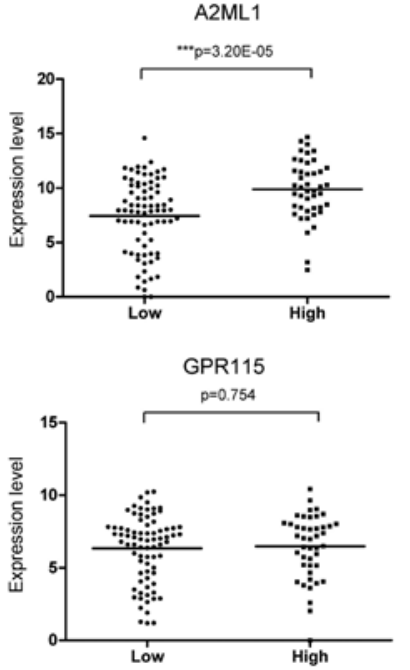
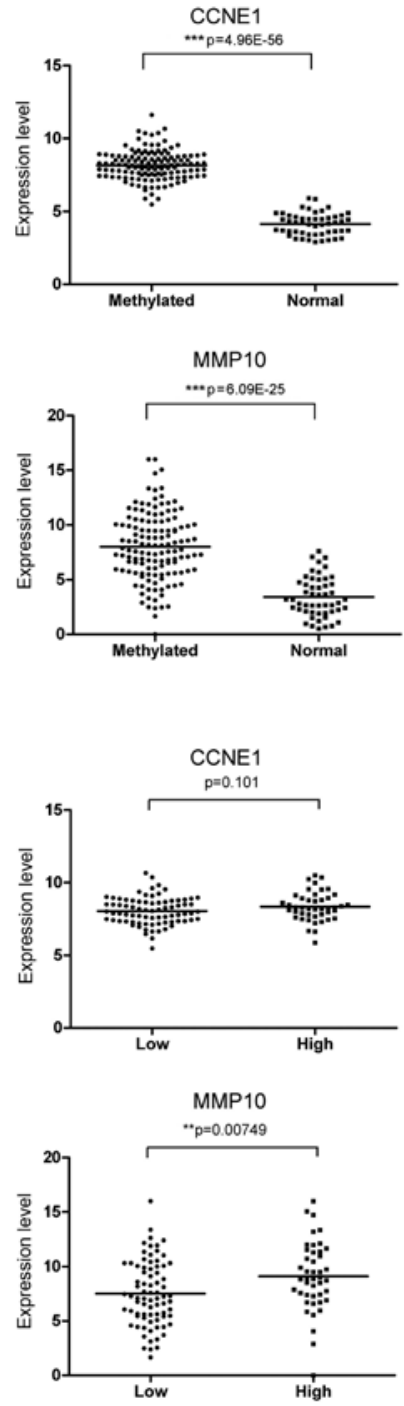
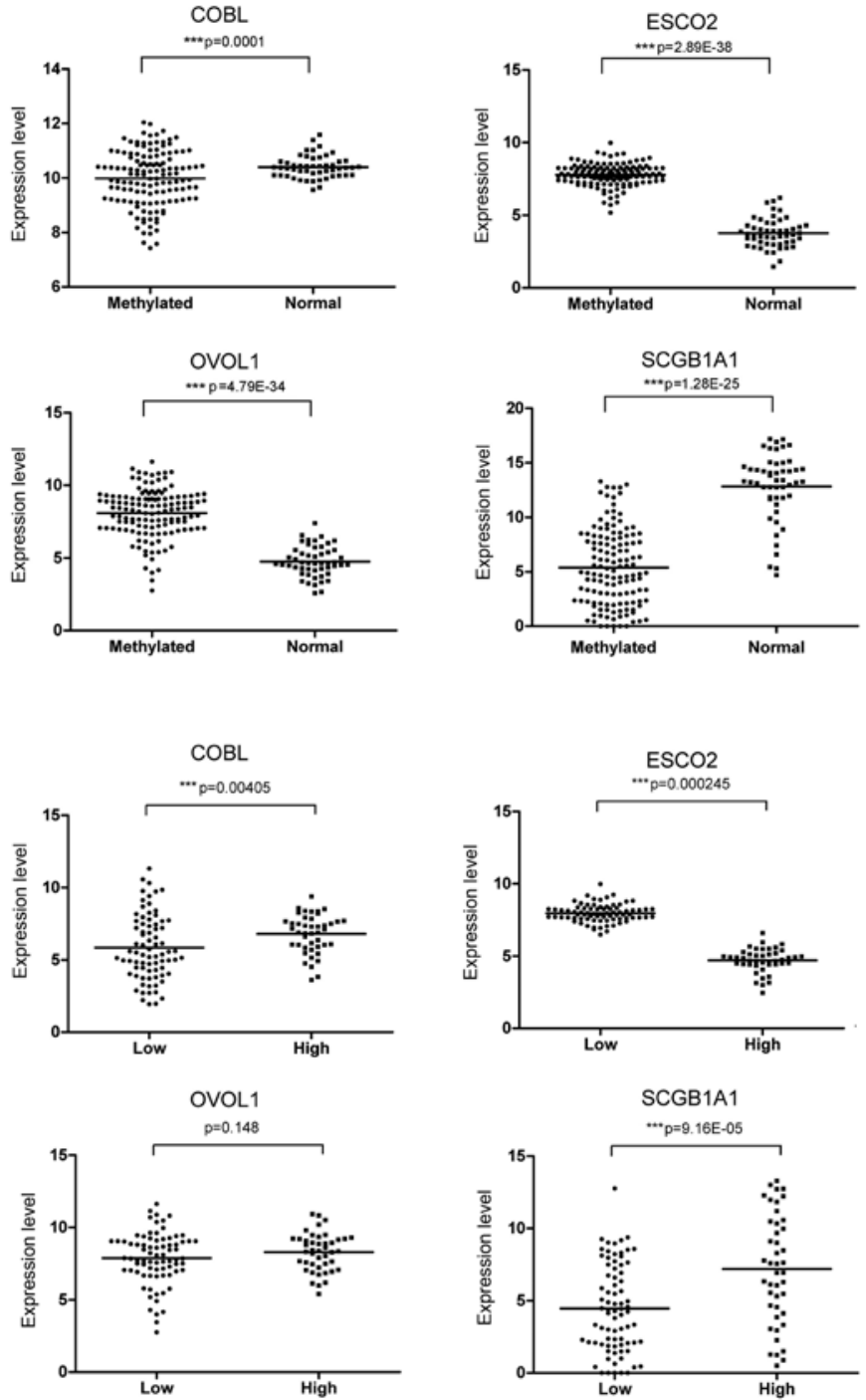

Figure 4. The expression values of 8 signature genes in different comparing sets. (A) High-methylation group compared to the normal group. (B) Low-risk group compared to the high-risk group. 
A a

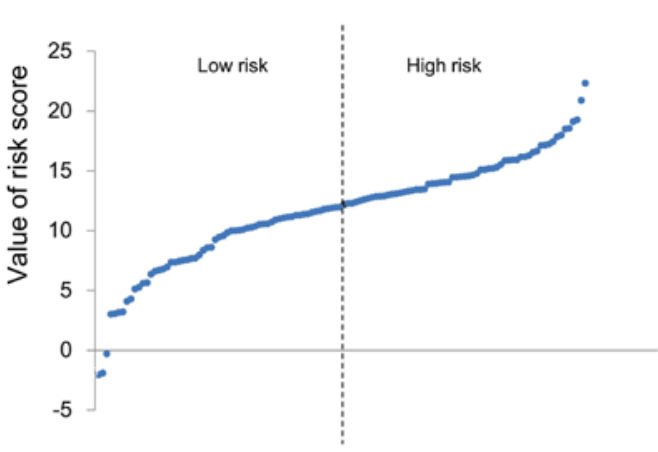

b
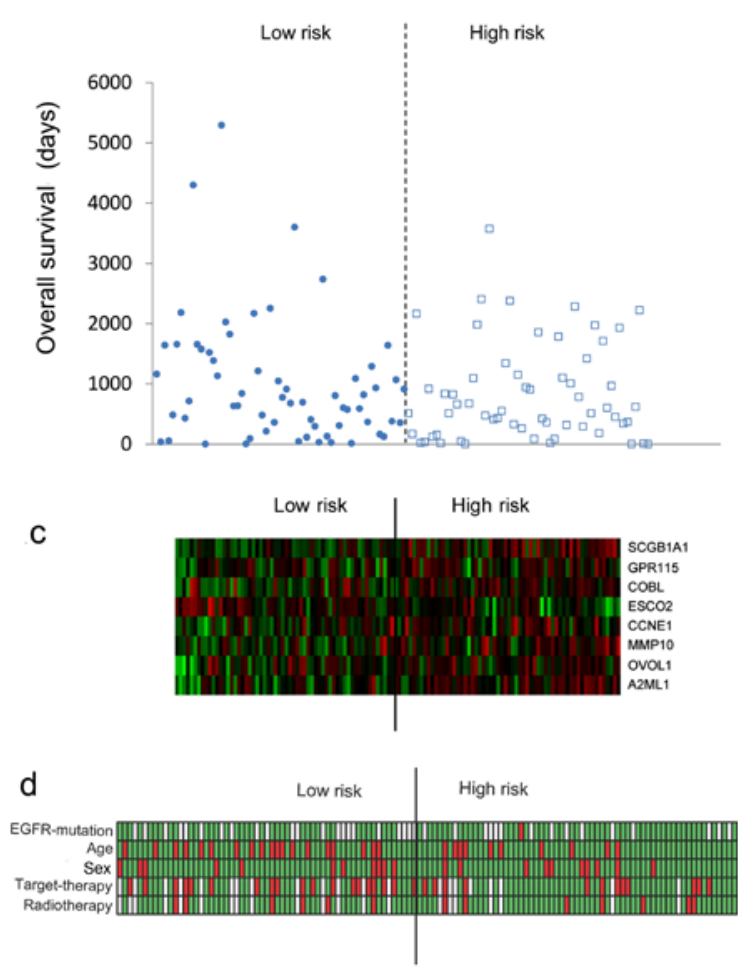

B a

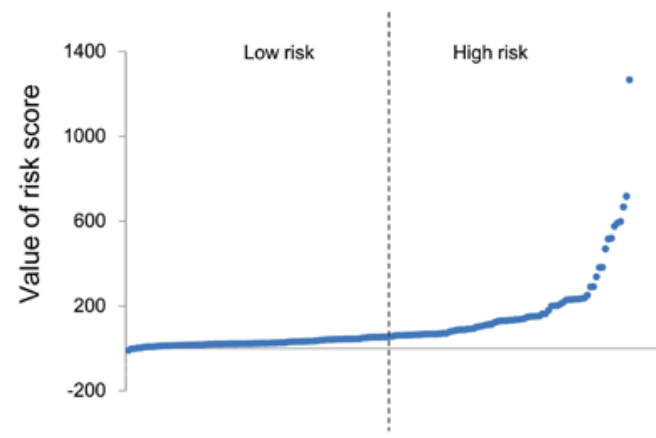

b
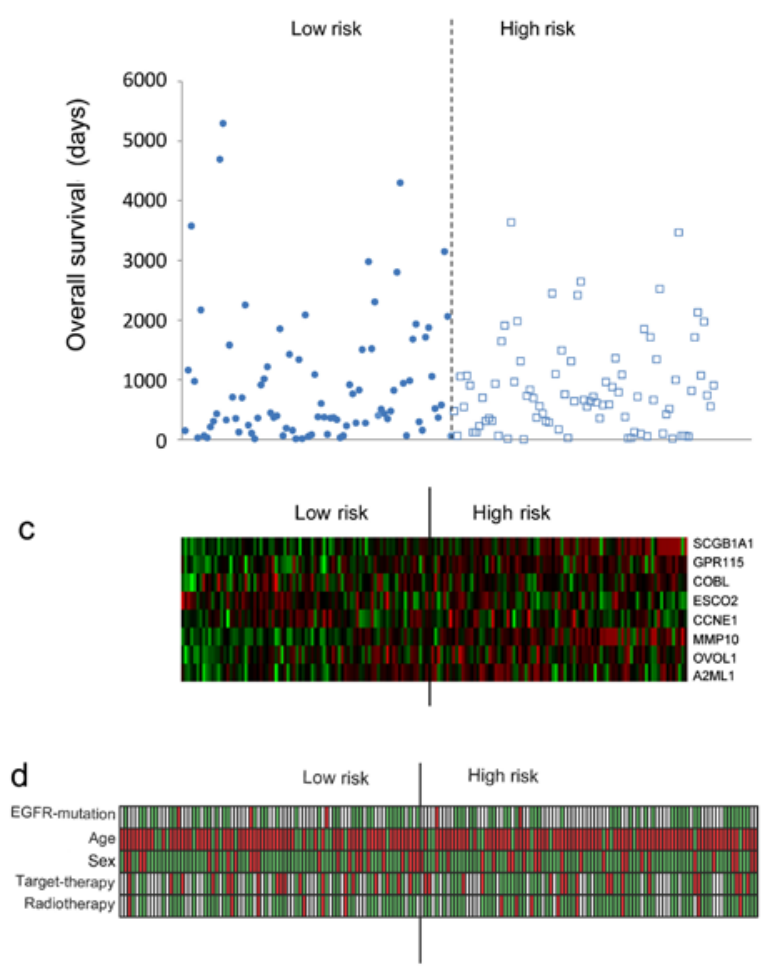

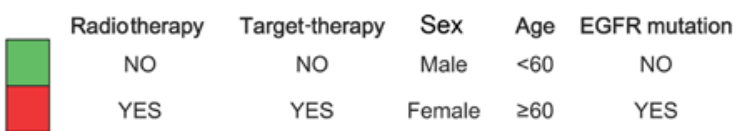

Figure 5. Clinical and molecular characteristics of patients with high and low risk of lung squamous cell carcinoma driven by TRIM58 methylation in (A) the training set and (B) the validation set. (A-a and B-a) Risk score. (A-b and B-b) Overall survival days. (A-c and B-c) Expression value of 8 signature genes. (A-d and B-d) Clinical information of the corresponding samples.

Table IV. Univariate and multivariate cox regression analysis of prognosis between samples and clinical information in the training dataset.

\begin{tabular}{|c|c|c|c|c|}
\hline \multirow[b]{2}{*}{ Variables } & \multicolumn{2}{|c|}{ Univariate cox } & \multicolumn{2}{|c|}{ Multivariable cox } \\
\hline & P-value & $\mathrm{HR}(95 \% \mathrm{CI})$ & P-value & $\mathrm{HR}(95 \% \mathrm{CI})$ \\
\hline Age (years) & 0.519 & $1.41(0.49-2.06)$ & & \\
\hline Sex & 0.703 & $1.19(0.48-2.91)$ & & \\
\hline EGFR mutation & 0.934 & $0.921(0.722-1.006)$ & & \\
\hline Radiotherapy & 0.0049 & $0.67(0.22-2.05)$ & 0.0739 & $0.605(0.412-0.72)$ \\
\hline Targeted-therapy & 0.0188 & $1.06(0.47-2.42)$ & 0.0341 & $0.871(0.388-0.957)$ \\
\hline Risk score & 0.0393 & $1.105(1.005-1.22)$ & 0.0121 & $1.122(1.026-1.228)$ \\
\hline
\end{tabular}

HR, hazard ratio; CI, confidence interval. 
A a

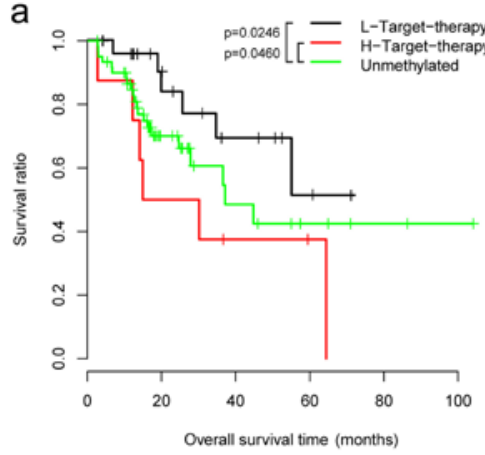

$\mathrm{B}$ a

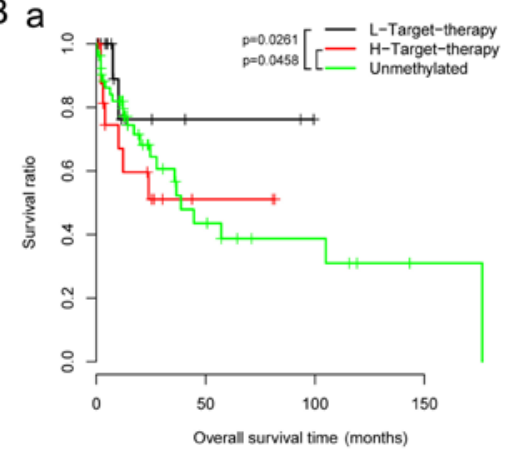

b
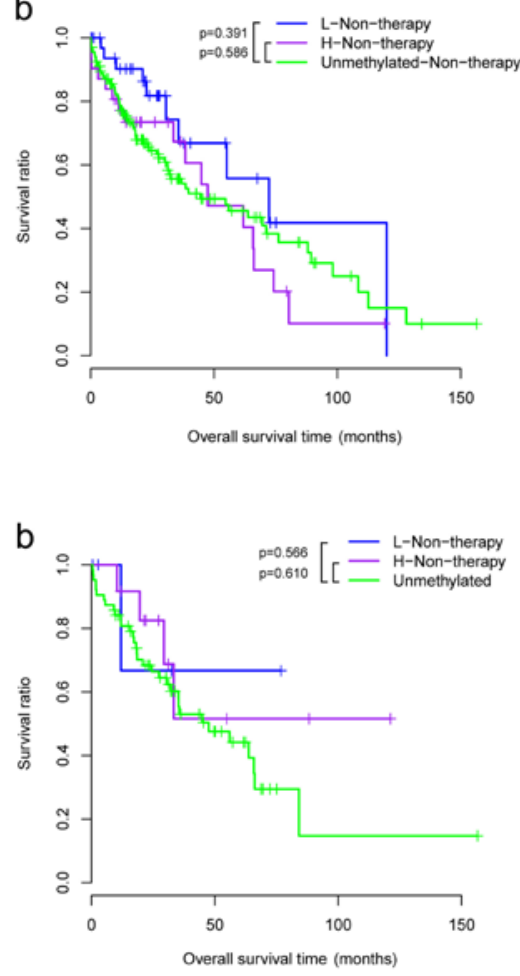
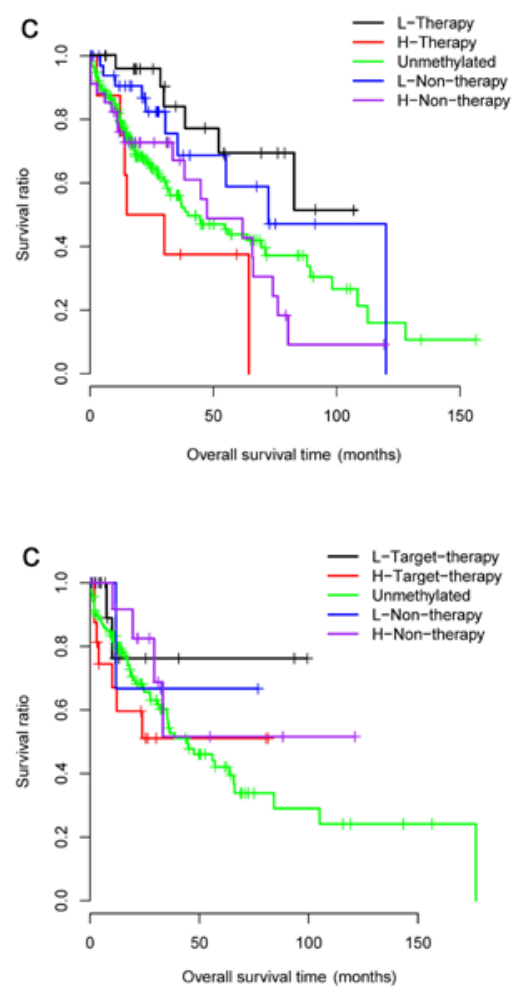

Figure 6. Kaplan-Meier (KM) survival curve of targeted therapy and risk score in the samples of the (A) training dataset and (B) validation dataset. (A-a and B-a) KM curves of targeted-treatment samples; (A-b and B-b) KM curves of non-targeted-treatment samples. (A-c and B-c) KM curves of both treated and untreated samples. H-Target-therapy, high-risk and target-treated samples (red curve); L-Target-therapy, low risk and target-treated samples (black curve) unmethylated, low methylation sample (green curve); H-Non-therapy, high risk and without target-treatment samples (blue curve); L-Non-therapy, high risk and without target-treatment samples (purple curve).

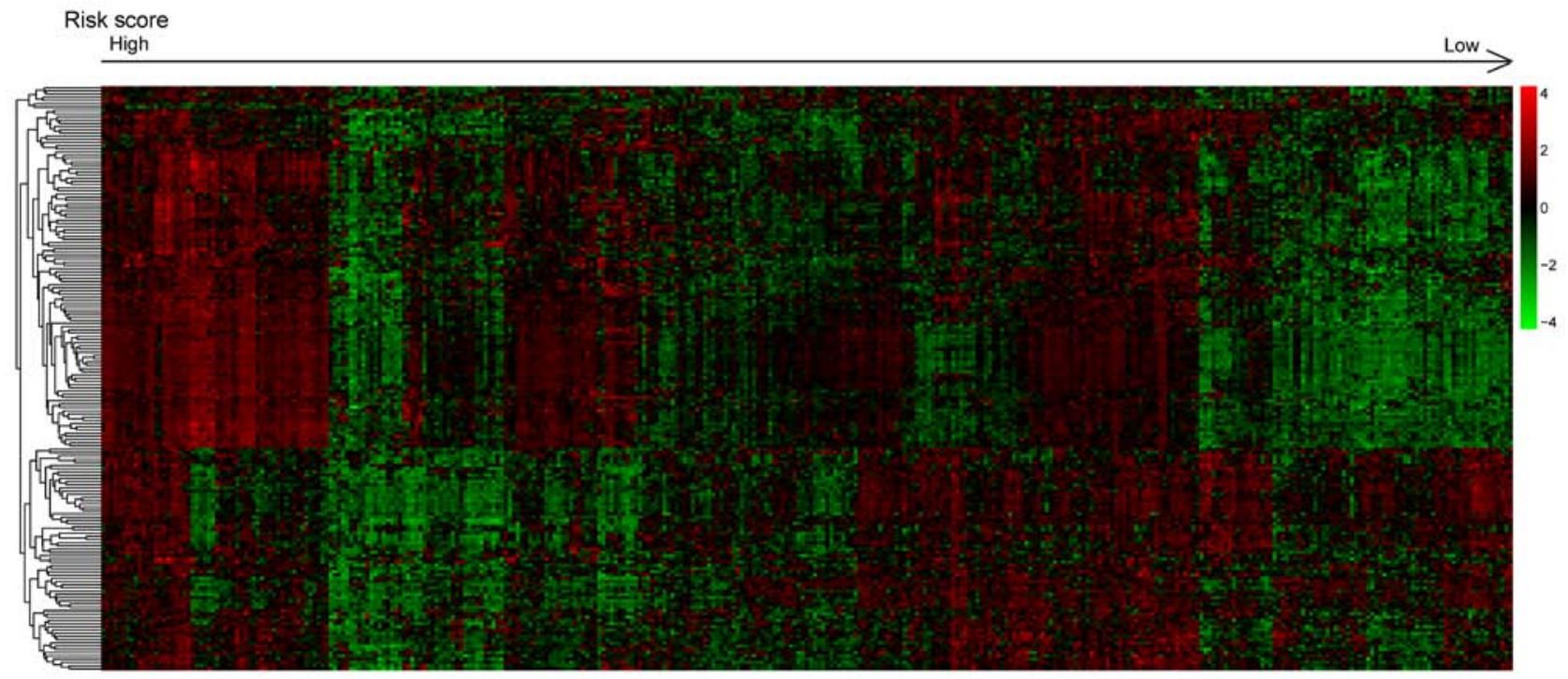

Figure 7. The hierarchical clustering chart of genes with significantly different expression level between the high- and the low-risk group in the training dataset.

the high-risk group was significantly lower than that of the low-risk group.

Functional enrichment analysis of genes associated with different prognosis. Totally, 181 differentially expressed genes between the high-risk group and the low-risk group in the training dataset were obtained with an FDR value of $<0.05$. The hierarchical clustering chart analysis was performed based on gene expression level (Fig. 7). GO function and KEGG pathway enrichment analysis of these genes were performed, (Fig. 8). Upregulated genes were mainly related to the functions of epithelial cell development, differentiation and keratinization, respiratory system development and immune response, while downregulated genes were mainly related to inorganic anion transport, (cellular) ion homeostasis and (cellular) chemical homeostasis. A total of 13 pathways were 


\section{A}

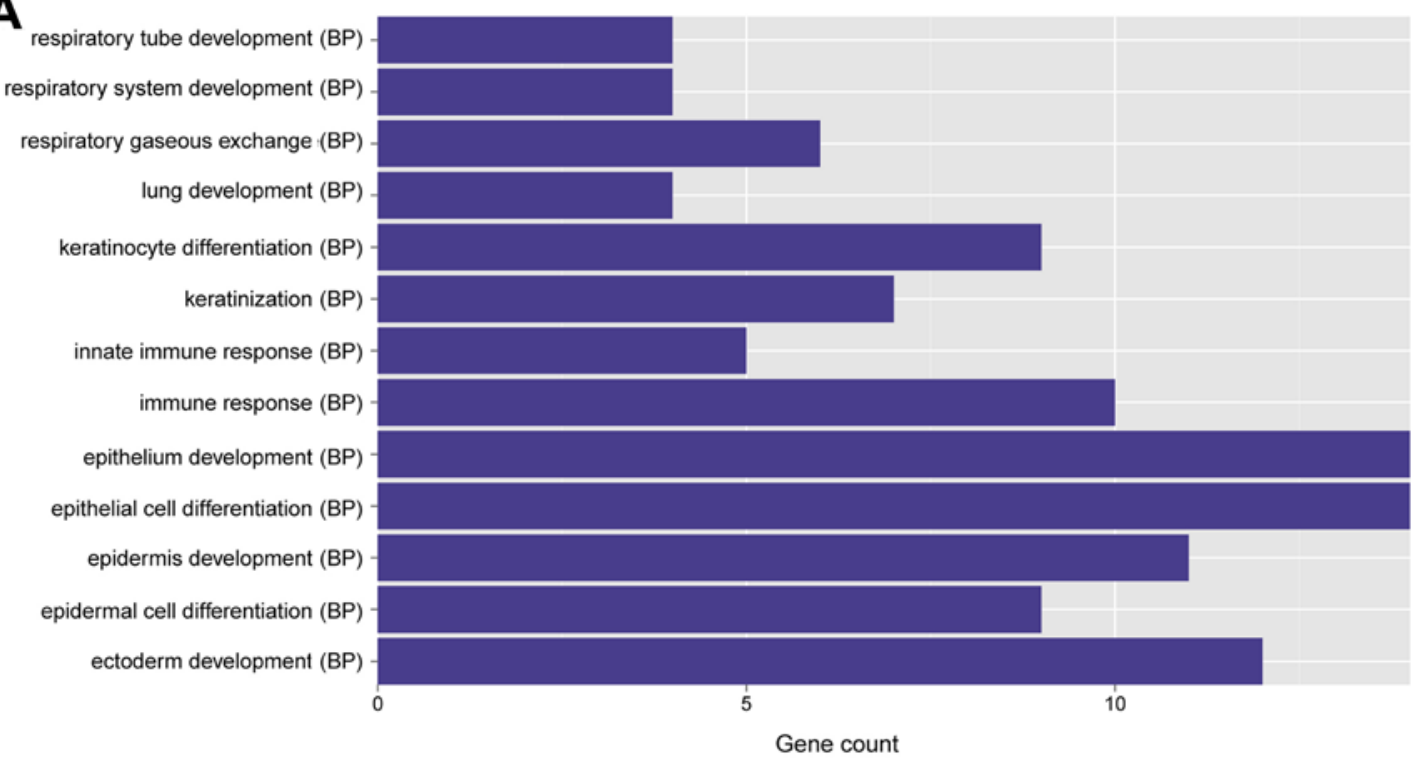

B

monovalent inorganic cation homeostasis (BP)

ion transport (BP)

ion homeostasis (BP)

inorganic anion transport (BP)

epithelium development (BP)

epithelial cell differentiation (BP)

chloride transport (BP)

chemical homeostasis (BP)

cellular ion homeostasis (BP)

cellular chemical homeostasis (BP)

cation homeostasis (BP)

anion transport (BP)

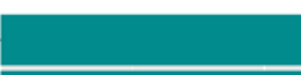

Gene coun

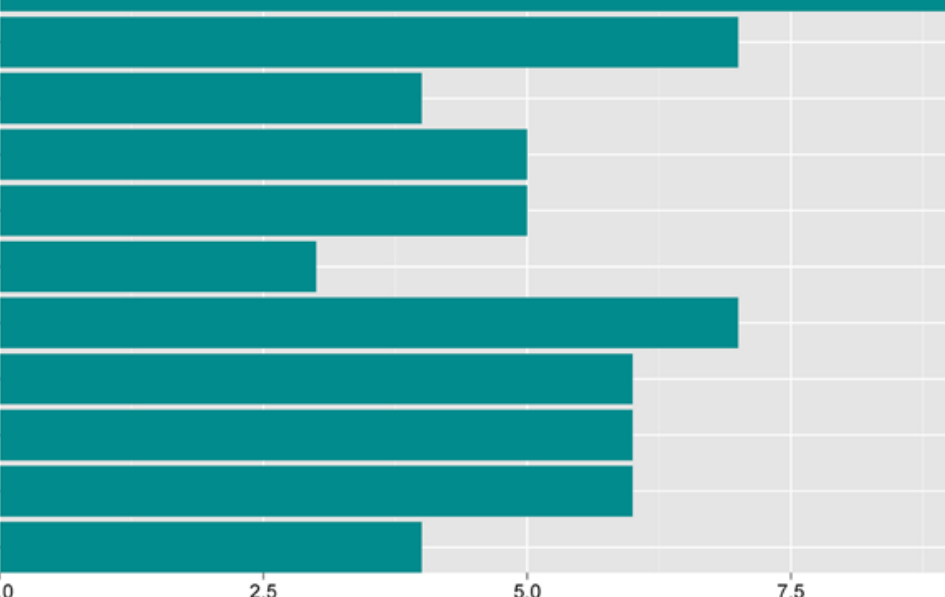

Gene count

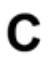

hsa05414: dilated cardiomyopathy

hsa05412: arrhythmogenic right ventricular cardiomyopathy (ARVC)

hsa05410: hypertrophic cardiomyopathy ( $\mathrm{HCM}$ )

hsa04610: complement and coagulation cascades

hsa02010:ABC transporters

hsa00982: drug metabolism

hsa00980:metabolism of xenobiotics by cytochrome P450

hsa00830: retinol metabolism

hsa00591: linoleic acid metabolism

hsa00590: arachidonic acid metabolism

hsa00350: tyrosine metabolism

hsa00071: fatty acid metabolism

hsa00010: glycolysis / gluconeogenesis

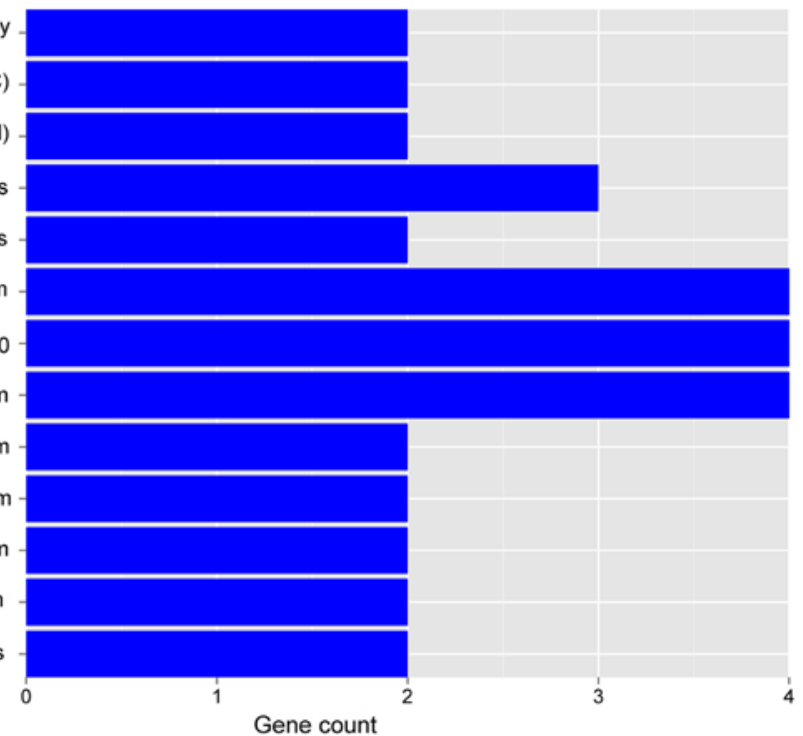

Figure 8. Functional enrichment analysis of genes associated with different prognosis. (A) Significant GO functions of genes with significantly upregulated expression. (B) Significant GO function analysis of genes with significantly downregulated expression. (C) KEGG pathway analysis of genes associated with different prognosis. 
enriched, such as drug metabolism, metabolism of xenobiotics by cytochrome P450, retinol metabolism and complement and coagulation cascades.

\section{Discussion}

Aberrant promoter island methylation of tumor suppressor genes has been established as a common epigenetic mechanism underlying the pathogenesis of human cancers, and may be used as diagnostic marker for tumorigenesis (5-7). In the present study, we tried to use the large quantities of mRNA-Seq data in lung squamous cell carcinoma patients published in cBioPortal database (http://cbioportal.org) to screen out candidate genes related to the methylation of TRIM58/cg26157385. Compared with the other large-scale cancer genomic projects, such as The Cancer Genome Atlas (TCGA) and the International Cancer Genome Consortium (ICGC) $(19,20)$, the cBioPortal for Cancer Genomics was specifically designed to lower the barriers of access to the complex datasets and thereby accelerate the translation of genomic data into new biological insights, therapies and clinical trials $(20,21)$. Based on the genomic data types integrated by cBioPortal, which include somatic mutations, DNA copy-number alterations, mRNA and microRNA expression, DNA methylation, protein abundance and phosphoprotein abundance, users achieved the exploration of multidimensional cancer genomic data and biological pathway, survival analysis, analysis of mutual exclusivity between genomic alterations, selective data download, programmatic access and publication-quality summary visualization (20). The present study was performed using 370 squamous cell lung carcinoma samples in the training dataset with methylation sequencing information and mRNA expression profiling data, and 178 squamous cell lung carcinoma samples in the validation dataset with mRNA expression profiling data. According to the DNA methylation $\beta$-values of TRIM58/cg26157385, the 370 samples in the training dataset were divided into 133 high-methylation samples and 237 low-methylation samples.

Significantly different expression genes, especially markedly upregulated or downregulated ones, often help reveal the mechanisms of various biological progresses and are usually preferred potential candidate genes for researchers to study. In the present study, 527 and 449 significantly different expression genes were gained in the high-methylation samples and low-methylation samples, respectively, and 629 significantly differentially expressed genes related to TRIM58 methylation were gained after the extraction of intersection between the two sets of differentially expressed genes. As a candidate tumor suppressor and a novel methylated gene $(7,10,12)$, aberrant inactivation of TRIM58 consequent to $\mathrm{CpG}$ island hyper-methylation may stimulate the early carcinogenesis of lung adenocarcinoma, and furthermore, TRIM58 methylation may be a possible early diagnostic and epigenetic therapeutic target in lung adenocarcinoma (7). Therefore, the association between the significantly differentially expressed genes related to TRIM58 methylation and lung cancer should be documented by combining with clinical information. After integration with the corresponding survival information for 347 out of 370 samples with squamous cell lung carcinoma, a total of 183 genes significantly associated with prognosis were gained using the Cox regression analysis of the survival package, and eight genes including A2ML1, GPR115, MMP10, CCNE1, ESCO2, COBL, OVOL1 and SCGB1A1 were selected as candidate signature genes.

The eight candidate signature genes were divided into four groups. The first group included three genes encoding enzyme and protease inhibitors, including MMP10, GPR115 and A2ML1. MMPs, a group of enzymes that are collectively capable of cleaving all components of the extracellular matrix, are involved in the epithelial migration, neoangiogenesis, matrix degradation and formation, among which, MMP10 was potential oral cancer marker (22-24). GPR115 is a member of the adhesion $\mathrm{G}$ protein-coupled receptors (GPCR) family, which are membrane-bound receptors with long $\mathrm{N}$-terminus, and several adhesion GPCRs are known to have important physiological functions in CNS development and immune system response mediated by large cell surface ligands, however, there has not been discovered a function for GPR115 (25). A2ML1 is a kind of protease inhibitor belonging to the alpha-macroglobulin superfamily and displays a unique trap mechanism of inhibition, by which the A2M inhibitor undergoes a major conformational change upon its cleavage by a protease, thereby trapping the protease and blocking it from subsequent substrate binding $(26,27)$. The second group included two genes, CNE1 and ESCO2, related to gene amplification. Gene amplification represents one of the molecular genetic hallmarks of human cancer and elucidating the molecular mechanisms of how amplified genes initiate and maintain malignant phenotypes and drive tumor progression was fundamental for understanding the molecular etiology of human cancer and its therapeutic implications (28). CCNE1 is the most frequent amplified gene in ovarian serous carcinomas and its gene amplification is related to poor survival and potential therapeutic target in ovarian cancer, and therefore, CCNE1-targeted therapy may benefit ovarian cancer patients with CCNE1 amplification $(28,29)$. ESCO2 gene encoded a protein which may have acetyltransferase activity and be required for the establishment of sister chromatid cohesion during the $\mathrm{S}$ phase of the cell cycle, and furthermore, it may function in transcription repression through modulation of the chromatin structure (30). Transcriptional control plays a key role in regulating epidermal proliferation and differentiation, and several transcription factors are known to regulate the balance between the mesenchymal to epithelial transition and the opposite program, such as COBL and OVOL1 in the third group (31-34). COBL is a WH2 domain-based actin nucleator, which has been demonstrated to play a critical role in dendrite formation and dendritic arborisation, and its spatial control is indispensable for proper establishment and plasticity of cell morphology $(31,34)$. OVOL1, encoding a zinc finger protein homologous to Drosophila melanogaster Ovo, is expressed in embryonic epidermal progenitor cells that are transiting from proliferation to terminal differentiation and regulates the growth arrest of embryonic epidermal progenitor cells and suppresses c-myc transcription $(32,33)$. There was one gene in the last group, SCGB1A1, an anti-inflammatory protein predominantly expressed by Clara cells in the lung, and a certain number of results indicated that low SCGB1A1 level may play a key role in the pathophysiology of asthma $(35,36)$. 
As MMP10, CNE1, ESCO2, COBL and OVOL1 had been reported to be related to cancer and other human lung diseases, we had reason to believe that the 8 candidate genes identified in the present study may be potential factors related to squamous cell lung carcinoma. Further validation of these 8 prognostic genes associated with TRIM58 methylation were performed using risk score, clinical and molecular characteristics of patients in the training dataset. The reliability of the prognostic discrimination system was further validated in an independent dataset. The present study provided potential diagnosis markers for the clinical diagnosis of lung squamous cell carcinoma and was helpful to explore the possible pathogenesis of lung squamous cell carcinoma.

Functional annotations of the significantly different expression genes according to GO and KEGG databases would provide ample numbers of candidate genes and more information about the pathogenesis of lung squamous cell carcinoma. In the present study, upregulated genes were mainly related to epithelial cell development, differentiation and keratinization, respiratory system development and immune response, while downregulated genes were mainly related to inorganic anion transport, (cellular) ion homeostasis and (cellular) chemical homeostasis. This indicated that the proliferation and differentiation of epidermal cells in lung squamous cell carcinoma patients were abnormal and the homeostasis was disturbed, and these findings were consistent with the histological changes of squamous cell lung carcinoma $(32,33)$. Further KEGG pathway analysis demonstrated that these genes were mainly involved in $13 \mathrm{KEGG}$ pathways. The expression alternation of genes involved in 8 metabolism related pathways in the present study again indicated the abnormal metabolism in lung squamous cell carcinoma patients, and this may give a clue for further clarifying the pathogenesis of lung squamous cell carcinoma.

It should be noted that the present study is an extensive bioinformatic study based on published data. The results of these studies should also be further validated in in vitro or in vivo models. We hope that these useful results will help other researchers perform relevant studies.

In conclusion, our data provided a comprehensive bioinformatic analysis of A2ML1, CCNE1, COBL, ESCO2, GPR115, MMP10, OVOL1 and SCGB1A1 as well as their corresponding pathways which may be involved in lung squamous cell carcinoma using two independent datasets. The results indicated that all of them were significantly related to the methylation of TRIM58/cg26157385 and treatment of lung squamous cell carcinoma. Therefore, these genes may be used as potential diagnostic markers and the present study would be helpful to elucidate the influence of TRIM58/cg26157385 methylation on lung cancer prognosis.

\section{Acknowledgements}

Not applicable.

\section{Funding}

The present study was supported by the general program of Wuxi municipal health and family planning commision (MS201715).

\section{Availability of data and materials}

The datasets used during the present study are available from the corresponding author upon reasonable request.

\section{Authors' contributions}

WZ conceived and designed the study. QC collected and pretreated the data, WQ, DJ and HL performed the data analysis. QC and WQ wrote the manuscript. WZ, QC, and HL reviewed and edited the manuscript. All authors read and approved the manuscript and agree to be accountable for all aspects of the research in ensuring that the accuracy or integrity of any part of the work are appropriately investigated and resolved.

\section{Ethics approval and consent to participate}

Not applicable.

\section{Consent for publication}

Not applicable.

\section{Competing interests}

The authors declare that they have no competing interests.

\section{References}

1. Chen W, Zheng R, Baade PD, Zhang S, Zeng H, Bray F, Jemal A, Yu XQ and He J: Cancer statistics in China, 2015. CA Cancer J Clin 66: 115-132, 2016.

2. Siegel RL, Miller KD and Jemal A: Cancer statistics, 2016. CA Cancer J Clin 66: 7-30, 2016.

3. Wistuba II, Behrens C, Milchgrub S, Bryant D, Hung J, Minna JD and Gazdar AF: Sequential molecular abnormalities are involved in the multistage development of squamous cell lung carcinoma. Oncogene 18: 643-650, 1999.

4. Cancer Genome Atlas Research Network: Comprehensive genomic characterization of squamous cell lung cancers. Nature 489: 519-525, 2012.

5. Imoto I, Izumi H, Yokoi S, Hosoda H, Shibata T, Hosoda F, Ohki M, Hirohashi S and Inazawa J: Frequent silencing of the candidate tumor suppressor PCDH20 by epigenetic mechanism in non-small-cell lung cancers. Cancer Res 66: 4617-4626, 2006.

6. Izumi H, Inoue J, Yokoi S, Hosoda H, Shibata T, Sunamori M, Hirohashi S, Inazawa J and Imoto I: Frequent silencing of DBC1 is by genetic or epigenetic mechanisms in non-small cell lung cancers. Hum Mol Genet 14: 997-1007, 2005.

7. Kajiura K, Masuda K, Naruto T, Kohmoto T, Watabnabe M, Tsuboi M, Takizawa H, Kondo K, Tangoku A and Imoto I: Frequent silencing of the candidate tumor suppressor TRIM58 by promoter methylation in early-stage lung adenocarcinoma. Oncotarget 8: 2890-2905, 2017.

8. Napolitano LM and Meroni G: TRIM family: Pleiotropy and diversification through homomultimer and heteromultimer formation. IUBMB Life 64: 64-71, 2012.

9. Nisole S, Saieb SA and Saïb A: TRIM family proteins: Retroviral restriction and antiviral defence. Nat Rev Microbiol 3: 799-808, 2005.

10. Qiu X, Huang Y, Zhou Y and Zheng F: Aberrant methylation of TRIM58 in hepatocellular carcinoma and its potential clinical implication. Oncol Rep 36: 811-818, 2016.

11. Thom CS, Traxler EA, Khandros E, Nickas JM, Zhou OY, Lazarus JE, Silva AP, Prabhu D, Yao Y, Aribeana C, et al: Trim58 degrades dynein and regulates terminal erythropoiesis. Dev Cell 30: 688-700, 2014.

12. White DE, Rafalskametcalf IU, Ivanov AV, Corsinotti A, Peng H, Lee SC, Trono D, Janicki SM and Rauscher FJ III: The ATM substrate KAP1 controls DNA repair in heterochromatin: Regulation by HP1 proteins and Serine 473/824 phosphorylation. Mol Cancer Res 10: 401-414, 2012. 
13. Wang X, Zhu H, Snieder H, Su S, Munn D, Harshfield G, Maria BL, Dong Y, Treiber F, Gutin B and Shi H: Obesity related methylation changes in DNA of peripheral blood leukocytes. BMC Med 8: 87, 2010.

14. Ritchie ME, Phipson B, Wu D, Hu Y, Law CW, Shi W and Smyth GK: limma powers differential expression analyses for RNA-sequencing and microarray studies. Nucleic Acids Res 43: e47, 2015.

15. Bao ZS, Li MY, Wang JY, Zhang CB, Wang HJ, Yan W, Liu YW, Zhang W, Chen L and Jiang T: Prognostic value of a nine-gene signature in glioma patients based on mRNA expression profiling. CNS Neurosci Ther 20: 112-118, 2014.

16. Cheng W, Ren X, Cai J, Zhang C, Li M, Wang K, Liu Y, Han S and $\mathrm{Wu} A$ : A five-miRNA signature with prognostic and predictive value for MGMT promoter-methylated glioblastoma patients. Oncotarget 6: 29285-29295, 2015.

17. Zhang CB, Zhu P, Yang P, Cai JQ, Wang ZL, Li QB, Bao ZS, Zhang $\mathrm{W}$ and Jiang T: Identification of high risk anaplastic gliomas by a diagnostic and prognostic signature derived from mRNA expression profiling. Oncotarget 6: 36643-36651, 2015.

18. Yu G, Wang LG, Han Y and He QY: clusterProfiler: An R package for comparing biological themes among gene clusters. OMICS 16: 284-287, 2012

19. International Cancer Genome Consortium; Hudson TJ, Anderson W, Artez A, Barker AD, Bell C, Bernabé RR, Bhan MK, Calvo F, Eerola I, et al: International network of cancer genome projects. Nature 464: 993-998, 2010

20. Gao J, Aksoy BA, Dogrusoz U, Dresdner G, Gross B, Sumer SO, Sun Y, Jacobsen A, Sinha R, Larsson E, et al: Integrative analysis of complex cancer genomics and clinical profiles using the cBioPortal. Sci Signal 6: pl1, 2013.

21. Cerami E, Gao J, Dogrusoz U, Gross BE, Sumer SO, Aksoy BA, Jacobsen A, Byrne CJ, Heuer ML, Larsson E, et al: The cBio cancer genomics portal: An open platform for exploring multidimensional cancer genomics data. Cancer Discov 2: 401-404, 2012.

22. Okada A, Tomasetto C, Lutz Y, Bellocq JP, Rio MC and Basset P: Expression of matrix metalloproteinases during rat skin wound healing: Evidence that membrane type-1 matrix metalloproteinase is a stromal activator of pro-gelatinase A. J Cell Biol 137: 67-77, 1997.

23. Vaalamo M, Karjalainen-Lindsberg ML, Puolakkainen $\mathrm{P}$, Kere J and Saarialhokere U: Distinct expression profiles of stromelysin-2 (MMP-10), collagenase-3 (MMP-13), macrophage metalloelastase (MMP-12), and tissue inhibitor of metalloproteinases-3 (TIMP-3) in intestinal ulcerations. Am J Pathol 152: 1005-1014, 1998.

24. Yen CY, Chen CH, Chang CH, Tseng HF, Liu SY, Chuang LY, Wen $\mathrm{CH}$ and Chang HW: Matrix metalloproteinases (MMP) 1 and MMP10 but not MMP12 are potential oral cancer markers. Biomarkers 14: 244-249, 2009.
25. Haitina T, Olsson F, Stephansson O, Alsiö J, Roman E, Ebendal T, Schiöth HB and Fredriksson R: Expression profile of the entire family of Adhesion $\mathrm{G}$ protein-coupled receptors in mouse and rat. BMC Neurosci 9: 1-14, 2008

26. Galliano MF, ToulzaE, Gallinaro H,Jonca N, Ishida-Yamamoto A, Serre G and Guerrin M: A novel protease inhibitor of the alpha2-macroglobulin family expressed in the human epidermis. J Biol Chemist 281: 5780-5789, 2006.

27. Vissers LE, Bonetti M, Paardekooper Overman J, Nillesen WM, Frints SG, de Ligt J, Zampino G, Justino A, Machado JC, Schepens M, et al: Heterozygous germline mutations in A2ML1 are associated with a disorder clinically related to Noonan syndrome. Eur J Hum Genet 23: 317-324, 2015.

28. Nakayama K, Nakayama N, Jinawath N, Salani R, Kurman RJ, Shih IeM and Wang TL: Amplicon profiles in ovarian serous carcinomas. Int J Cancer 120: 2613-2617, 2007.

29. Nakayama N, Nakayama K, Shamima Y, Ishikawa M, Katagiri A, Iida K and Miyazaki K: Gene amplification CCNE1 is related to poor survival and potential therapeutic target in ovarian cancer. Cancer 116: 2621-2634, 2010.

30. Kim BJ, Kang KM, Jung SY, Choi HK, Seo JH, Chae JH, Cho EJ, Youn HD, Qin J and Kim ST: Esco2 is a novel corepressor that associates with various chromatin modifying enzymes. Biochem Biophys Res Commun 372: 298-304, 2008.

31. Ahuja R, Pinyol R, Reichenbach N, Custer L, Klingensmith J, Kessels MM and Qualmann B: Cordon-bleu is an actin nucleation factor and controls neuronal morphology. Cell 131: 337-350, 2007.

32. Nair M, Teng A, Bilanchone V, Agrawal A, Li B and Dai X: Ovoll regulates the growth arrest of embryonic epidermal progenitor cells and represses c-myc transcription. J Cell Biol 173: 253-264, 2006.

33. Roca H, Hernandez J, Weidner S, McEachin RC, Fuller D, Sud S, Schumann T, Wilkinson JE, Zaslavsky A, Li H, et al: Transcription factors OVOL1 and OVOL2 induce the mesenchymal to epithelial transition in human cancer. PloS One 8: e76773, 2013

34. Schwintzer L, Koch N, Ahuja R, Grimm J, Kessels MM and Qualmann B: The functions of the actin nucleator Cobl in cellular morphogenesis critically depend on syndapin I. Embo J 30: 3147-3159, 2011.

35. Nie W, Xue C, Chen J and Xiu Q: Secretoglobin 1A member 1 (SCGB1A1)+38A/G polymorphism is associated with asthma risk: A meta-analysis. Gene 528: 304-308, 2013.

36. Peri A, Cordella-Miele E, Miele L and Mukherjee AB: Tissue-specific expression of the gene coding for human Clara cell $10-\mathrm{kD}$ protein, a phospholipase A2-inhibitory protein. J Clin Invest 92: 2099-2109, 1993. 